hep-ph/9712238

\title{
An Updated Analysis on Atmospheric Neutrinos*
}

\author{
M. C. Gonzalez-Garcia ${ }^{1.2}$, H. Nunokawa ${ }^{1}$, O. Peres ${ }^{1}$, \\ T. Stanev ${ }^{3}$ and J. W. F. Valle ${ }^{1}$ \\ ${ }^{1}$ Instituto de Física Corpuscular - C.S.I.C. \\ Departament de Física Teòrica, Universitat de València \\ 46100 Burjassot, València, Spain \\ ${ }^{2}$ Instituto de Física Teórica, Universidade Estadual Paulista \\ Rua Pamplona 145, 01405-900 São Paulo, Brazil \\ ${ }^{3}$ Bartol Research Institute, University of Delaware \\ Newark, Delaware 197161, USA
}

\begin{abstract}
We have reanalysed the atmospheric neutrino data including new results from SuperKamiokande and Soudan-II experiments, under the assumption of two-flavor neutrino oscillation. We present the allowed region of oscillation parameters for the $\nu_{\mu} \rightarrow \nu_{\tau}$ channel. In performing this re-analysis we also take into account some recent theoretical improvements in the flux calculations.
\end{abstract}

The atmospheric neutrino anomaly [1] observed in Kamiokande [2] and IMB [3] has recently been confirmed by new results from Super-Kamiokande [4] and Soudan-II [5]. The significance of the problem is usually expressed in terms of the double ratio, $R \equiv\left(N_{\mu} / N_{e}\right)_{\text {observed }} /\left(N_{\mu} / N_{e}\right)_{\text {predicted }}$, where $N_{\mu}$ and $N_{e}$ stand for the number of $\mu$ and $e$-like events, respectively. All the experiments, except for Frejus [6] and NUSEX [7], are observing the deficit in the double ratio, $R \simeq 0.6$.

The most natural and simplest solution to this problem is given by the two-flavor neutrino oscillation, either due to $\nu_{\mu}-\nu_{\tau}$ or $\nu_{\mu}-\nu_{e}$ channel, the latter being most likely disfavoured by the recent result from the $\mathrm{CHOOZ}$ experiment [8]. Under this assumption, we have re-analysed the atmospheric neutrino data coming from Kamiokande [2], IMB [3], Frejus [6], Nusex [7], as well as those new (preliminary) ones coming from Super-Kamiokande [4] and Soudan-II [5]. In our analysis we have used one of the latest calculations of atmospheric neutrino flux [9] which, in general, depends on energy and direction of neutrinos and solar activity, as well as experimental site, due to the geomagnetic effect. In our analysis we have also included the effect of production point distribution of neutrinos [10], which should, in principle, be taken into account in the determination of the oscillation parameters.

We have performed a detailed $\chi^{2}$ analysis, treating separately the $\mu$ and $e$-like data, but taking into account the correlation of errors. This is better than using the double ratio $R$, due to its non-Gaussian nature, as suggested in ref. [11]. We present in Fig. 1 our (preliminary) results for the $\nu_{\mu}-\nu_{\tau}$ oscillation channel, in (a) for each experiment and in (b) for combined result. We notice that the allowed region is mostly determined by new results from Super-Kamiokande multi-GeV binned data. We obtained the best fit point around $\sin ^{2} 2 \theta \sim 1$ and $\Delta m^{2} \sim 10^{-3} \mathrm{eV}^{2}$. Let us note that the best fit value of $\Delta m^{2}$ is now lower than the one in the pre-Super-Kamiokande era.

*Talk given by H. Nunokawa (e-mail: nunokawa@flamenco.ific.uv.es) at Erice School on Nucl. Physics, 19th course "Neutrinos in Astro, Particle and Nuclear Physics", Erice, Italy, September 1997, to appear in the proceedings. 
More detailed description and discussion of the analysis, including the case for $\nu_{\mu}-\nu_{e}$ channel, will be given in ref. [12].
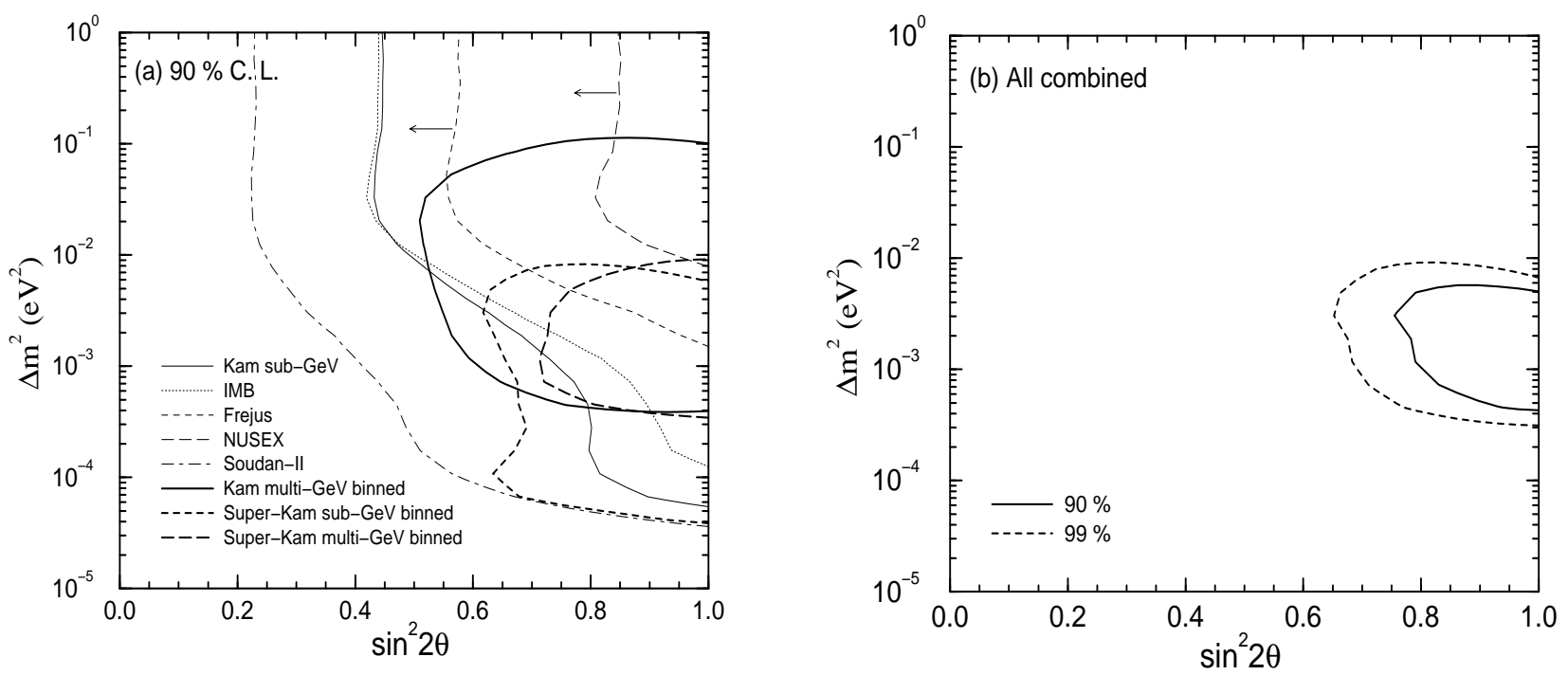

Fig. 1: In (a) we plot the individual contour for each experiment for $90 \%$ C. L. and in (b) we plot the combined results for 90 and $99 \%$ C. L. In (a) the regions right to the curves are allowed except for Frejus and NUSEX.

\section{References}

[1] For reviews, see for e.g., T. Kajita, in Physics and astrophysics of neutrinos, p. 559, ed. by M. Fukugita and A. Suzuki, Tokyo, Springer-Verlag, 1994; T. Stanev, Nucl. Phys. (Proc. Suppl) B 48 (1996) 165.

[2] Kamiokande Collaboration, H. S. Hirata et al., Phys. Lett. B205 (1988) 416 and Phys. Lett. B280, (1992) 146 ; Y. Fukuda et al., Phys. Lett. B335, (1994) 237.

[3] IMB Collaboration, D. Casper et al., Phys. Rev. Lett. 66, 2561 (1991); R. Becker-Szendy et al., Phys. Rev. D46, 3720 (1992).

[4] Super-Kamiokande Collaboration, Y. Totsuka, talk at the Lepton-Photon '97, August 1997, Hamburg, Germany; E. Kearns, talk at TAUP97, LNGS, Assergi, Italy, September 1997.

[5] Soudan-II Collaboration, T. Kafka, talk at Taup 97, LNGS, Assergi, Italy, September 1997.

[6] Fréjus Collaboration, Ch. Berger et al., Phys. Lett. B227, 489 (1989).

[7] NUSEX Collaboration, M. Aglietta et al., Europhys. Lett. 8 (1989) 611.

[8] M. Apollonio et al., CHOOZ Collaboration, preprint, hep-ex/9711002.

[9] V. Agrawal et al., Phys. Rev. D53, 1314 (1996); T.K.Gaisser and T.Stanev, in Proc. 24th ICRC, Vol. 1, p. 694 (Rome 1995).

[10] T. K. Gaisser and T. Stanev, preprint BRI-97-28, astro-ph/9708146.

[11] G. L. Fogli, E. Lisi and D. Montanino, Phys. Rev. D49 (1994) 3626; G. L. Fogli, E. Lisi, Phys. Rev. D52 (1995) 2775.

[12] M. C. Gonzalez-Garcia, H. Nunokawa, O. Peres, J.W.F. Valle, T. Stanev, in preparation. 